\title{
Synthesis of benzothienopyridopyrimidinones and benzothienopyrimidoisoquinolinone by microwave-assisted dehydrogenation of the corresponding tetrahydro derivatives
}

\author{
Agathe Begouin, Stéphanie Hesse,* and Gilbert Kirsch \\ Laboratoire d'Ingénierie Moléculaire et Biochimie Pharmacologique, Institut Jean Barriol, \\ Université Paul Verlaine - Metz, 1 Boulevard Arago, 57070 METZ Technopôle, France \\ E-mail: hesse@univ-metz.fr
}

Dedicated to Professor Guy Quéguiner on the occasion of his 70th birthday

\begin{abstract}
DDQ was employed as a dehydrogenation reagent to synthesize polyaromatic compounds from the corresponding tetrahydro derivatives. A microwave-assisted process allows shorter reaction times and easier purification as fewer degradation products were formed.
\end{abstract}

Keywords: Microwave, dehydrogenation, DDQ, benzothienopyridopyrimidinone

\section{Introduction}

Thienopyrimidines and thienopyrimidinones are found to exhibit a variety of biological activities, including anti-inflammatory, ${ }^{1}$ antimicrobial $^{2}$ and analgesic ${ }^{3}$ properties, inhibition of cancer cell proliferation, ${ }^{4}$ anti-angiogenic activities, ${ }^{5}$ antagonism of $\alpha 1$-adrenoceptors,${ }^{6}$ and antagonism of mGluR $1{ }^{7}$ Consequently, thienopyrimidines have become a well-sought privileged class of compounds in drug discovery programs.

Recently, we described the "one-pot" palladium-catalyzed cross-coupling/cyclization reaction of compound $\mathbf{1}$ with 2-aminopyridine derivatives or 1-aminoisoquinoline. ${ }^{8}$ Tetra- and pentacyclic compounds $\mathbf{2 - 4}$ were obtained in high yields (Scheme 1). 

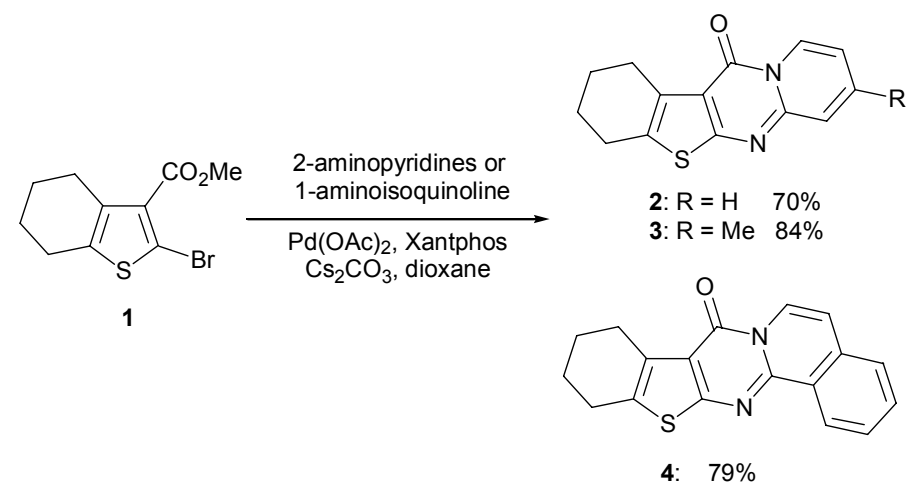

\section{Scheme 1}

On the other hand, planar polycyclic aromatic molecules are known to intercalate into double-stranded DNA. ${ }^{9}$ In 2006, Viola et al. ${ }^{10}$ described the induction of apoptosis by a photoexcited benzo[b]thieno[3,2-d]pyrido[1,2-a]pyrimidin-6-one I (Figure 1). Aiming to evaluate compounds $\mathbf{2 - 4}$, we decided to synthesize the benzo[b]thieno[2,3- $d]$ pyrido[1,2a]pyrimidin-12-ones II by aromatization of the latter compounds. We thought it would be interesting to compare the biological activities of the tetrahydro- derivatives and the corresponding fully aromatic compounds.
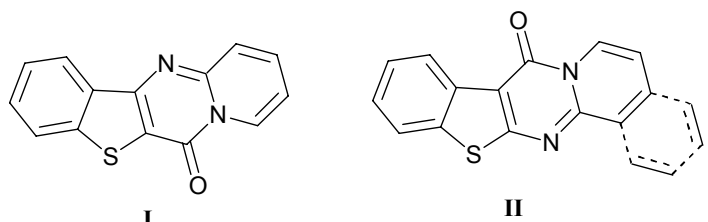

\section{Figure 1}

\section{Results and Discussion}

Many reagents can be employed to perform dehydrogenation reactions but we generally use $\mathrm{Pd} / \mathrm{C}$ or DDQ. ${ }^{11}$ The aromatization of compound $\mathbf{2}$ was first studied to establish the most effective conditions. No satisfying results were obtained when using $\mathrm{Pd} / \mathrm{C}$ as starting material was recovered together with some degradation products. (Table 1, entries 1-2) 
Table 1. Aromatization of compound 2 by heating

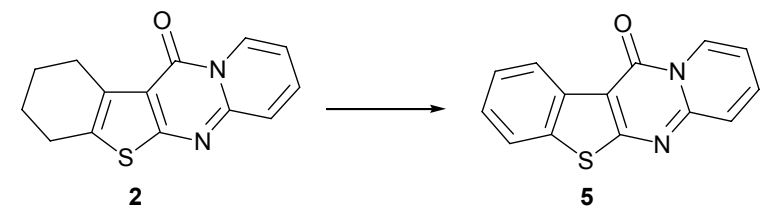

\begin{tabular}{clc}
\hline Entry & Conditions & Yield \\
\hline 1 & $20 \mathrm{~mol} \% \mathrm{Pd} / \mathrm{C}-o$-dichlorobenzene $-24 \mathrm{~h}-180^{\circ} \mathrm{C}$ & - \\
2 & $40 \mathrm{~mol} \% \mathrm{Pd} / \mathrm{C}-o$-dichlorobenzene $-72 \mathrm{~h}-220^{\circ} \mathrm{C}$ & - \\
3 & 2 equiv. DDQ - toluene $-21 \mathrm{~h}-$ reflux & - \\
4 & 3 equiv. DDQ $-o$-dichlorobenzene $-24 \mathrm{~h}-160^{\circ} \mathrm{C}$ & - \\
5 & 2.5 equiv. DDQ $-o$-dichlorobenzene $-4 \mathrm{~h}-190^{\circ} \mathrm{C}$ & $59 \%$ \\
6 & 3 equiv. DDQ $-o$-dichlorobenzene $-24 \mathrm{~h}-190^{\circ} \mathrm{C}$ & $36 \%$ \\
7 & 2.5 equiv. DDQ -2 -(2-butoxyethoxy)ethanol $-30 \mathrm{~min}-230^{\circ} \mathrm{C}$ & - \\
\hline
\end{tabular}

The use of DDQ as reagent provided the formation of 5, but 2.5 equivalents were needed for the reaction to reach completion. Moreover, the effect of temperature seems to be critical, as we did not obtain compound 5, either at reflux in toluene or by heating at $160^{\circ} \mathrm{C}$ in $o$ dichlorobenzene (Table 1, entries 3-4). Increasing the temperature to $190^{\circ} \mathrm{C}$ allowed the aromatization to be performed. The reaction time was also important, as we observed the appearance of degradation products after a few hours; compound $\mathbf{5}$ was then more difficult to isolate. The yield of 5 decreased from $59 \%$ to $36 \%$ when the reaction time was increased from $4 \mathrm{~h}$ to $24 \mathrm{~h}$. (Table 1, entries 5-6). We also tried a reaction at higher temperature for a shorter time (Table 1, entry 7) but only degradation products were obtained.

As microwave-assisted synthesis often enhances the reaction rate and allows shorter reaction times, we decided to perform the aromatization under microwave irradiation. We hoped that reducing the reaction time would reduce the formation of secondary products and improve the yield. Only a few publications ${ }^{12-14}$ have reported microwave-assisted dehydrogenation. Whereas Romagnoli and co-workers ${ }^{12}$ did not noticed any yield improvement (only a reduction of reaction time: 5 min vs 3 hours in conventional heating), Alvarez et al. ${ }^{13}$ observed that microwaveassisted aromatization of dihydroisoquinoline was superior.

Microwave-assisted reactions can be performed under two types of microwave control: the first one (standard control) is a "transposition" of classical heating conditions with a control of temperature and time (the microwave controls the irradiation power to maintain the fixed temperature). The second one corresponds to a power/time control with only an infrared measurement of the temperature reached in the mixture. We first tried dehydrogenation of compound 2 under standard control $\left(10 \mathrm{~min}\right.$ at $\left.180^{\circ} \mathrm{C}\right)$. After treatment and purification, compound 5 was obtained in $57 \%$ yield. (Scheme 2) 

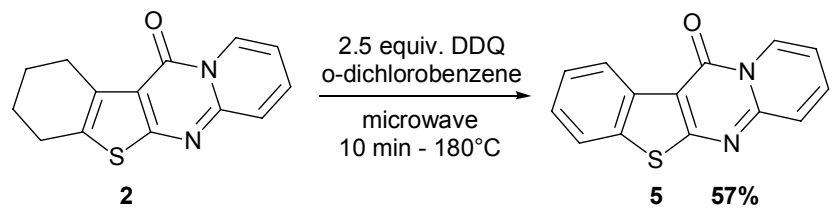

\section{Scheme 2}

Aiming to optimize reactions under microwave irradiation, we paid careful attention to each parameter. In this experiment, microwave irradiation of the reaction mixture for $4.5 \mathrm{~min}$ at a power between 110 and $300 \mathrm{~W}$ was used to reach the fixed temperature (run time); then the microwave adjusted the power to maintain the temperature at $180^{\circ} \mathrm{C}$ (hold time). Starting from $300 \mathrm{~W}$, it decreased to $35 \mathrm{~W}$ (Figure 2). In fact, the reaction mixture was irradiated for $14.5 \mathrm{~min}$ overall. As we obtained almost the same yield under both conditions (classical heating and microwave irradiation), this clearly showed that microwave-assisted dehydrogenation is a timesaving procedure.

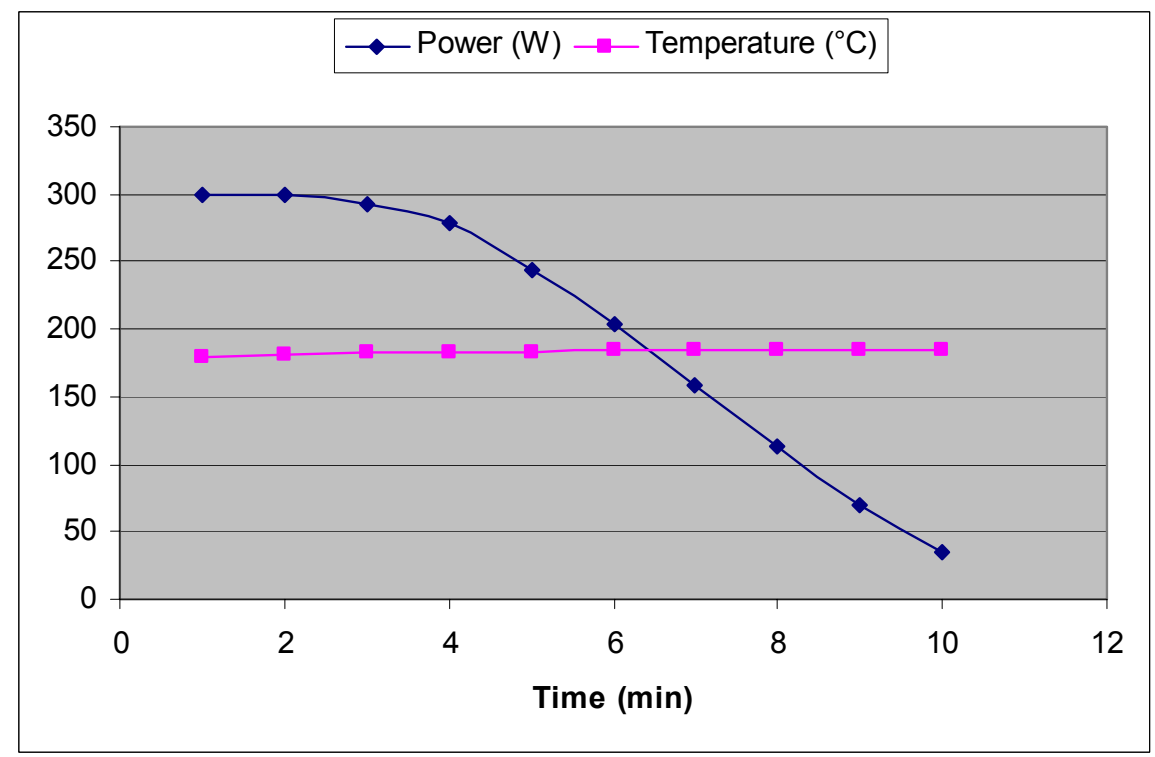

\section{Figure 2}

We then performed the same reaction in toluene at $110^{\circ} \mathrm{C}$ for 10 minutes. The fixed temperature was reached after 1 minute of run time; the evolution of the power is shown in figure 3. Unfortunately, starting material and degradation products were obtained but the expected compound has not been isolated. As in classical heating, a high temperature is necessary. 


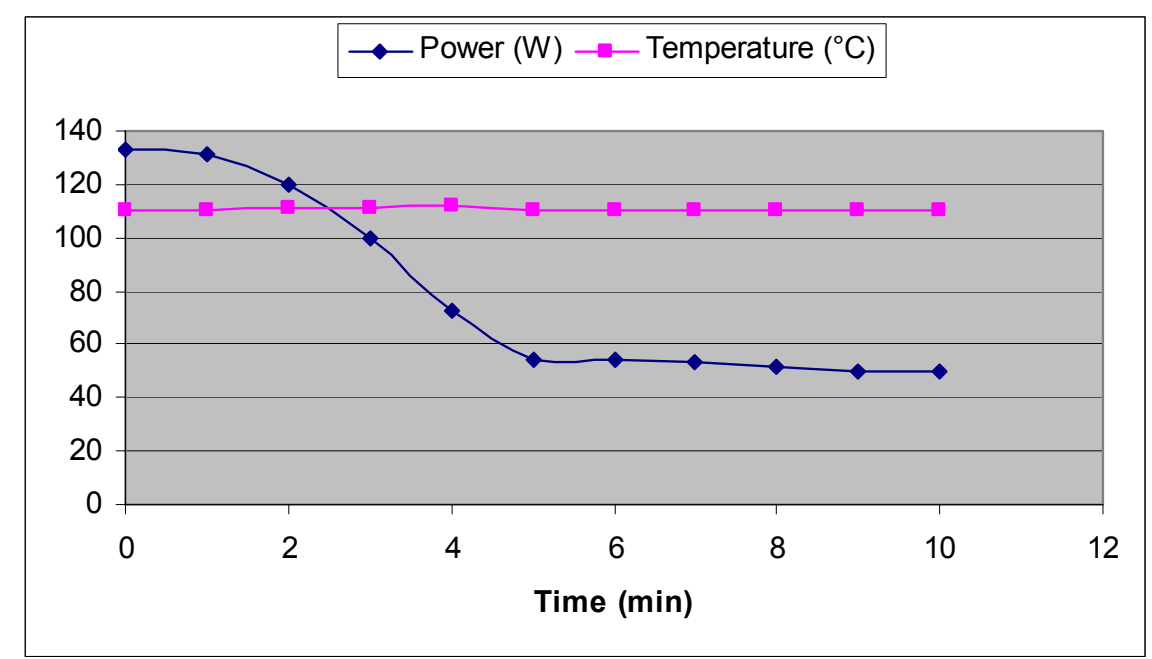

\section{Figure 3}

In order to see more clearly the impact of the power on the yield, we decided to work under power/time control: the reaction mixture was irradiated at $150 \mathrm{~W}$ for 5 minutes with $200^{\circ} \mathrm{C}$ as maximum temperature (if this temperature is reached, the system will cease to apply microwave energy). TLC showed that starting material was still present. So, two other attempts were made: a sample was irradiated at $150 \mathrm{~W}$ for 10 minutes and another at $300 \mathrm{~W}$ for 5 minutes; compound 5 was obtained in respectively $62 \%$ and $65 \%$ yield after purification. In both cases, the temperature reached is almost the same but working at higher power allows a shorter reaction time. (Figure 4).

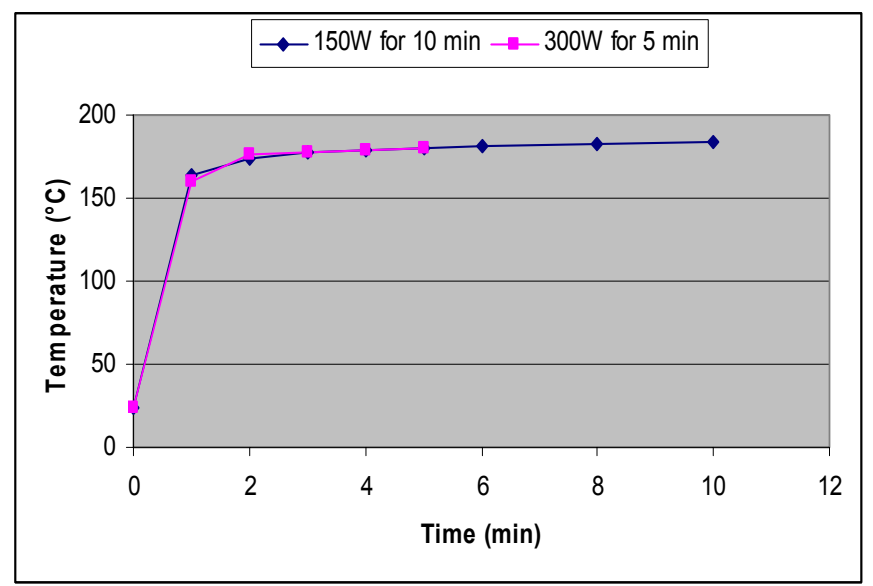

\section{Figure 4}

We then applied the last conditions as well as the classical conditions described before to compounds 3-4 (Table 2). The yields obtained in the microwave-assisted procedure were as good 
as or better than those obtained by classical heating. Having been successful using compounds 2-4, we decided to extend the dehydrogenation to other types of tetrahydro-derivatives prepared earlier in the laboratory. Results are presented in Table 2.

Table 2. Aromatization of tetrahydro derivatives under classical or microwave heating

$\begin{gathered}\text { Classical } \\ \text { heating }\end{gathered}$
$\begin{gathered}\text { Microwave } \\ \text { irradiation }\end{gathered}$

In conclusion, tetra- and pentacyclic fully aromatic compounds were obtained in good- to high yields by dehydrogenation of the corresponding tetrahydro derivatives using DDQ as reagent. The microwave-assisted procedure was proved to be superior, as only 5 minutes of reaction were needed and yields were generally superior.

\section{Experimental Section}

General Procedures. Melting points were determined on a Stuart SMP3 apparatus and are uncorrected. ${ }^{1} \mathrm{H}$ - and ${ }^{13} \mathrm{C}$ - spectra were recorded on an AC Bruker $250 \mathrm{MHz}$ spectrometer. IR spectra were recorded on a Perkin Elmer Spectrum BX. HRMS spectra were recorded on a Maldi-TOF MS Bruker Reflex.

General procedure for aromatization (conventional heating). A round bottom flask was charged with compounds $\mathbf{2 - 4}$ as starting materials, DDQ (2.5 equiv.) and $o$-dichlorobenzene as solvent $(5 \mathrm{~mL})$. The reaction mixture was stirred and heated at reflux for $4 \mathrm{~h}$. After the mixture had been cooled, $o$-dichlorobenzene was removed under vacuum, dichloromethane was added to the resulting solid, and this solution was filtered using Celite. The filtrate was evaporated under reduced pressure and compounds 5-9 were obtained after purification by column chromatography with $\mathrm{CHCl}_{3}$. 
General procedure for aromatization (microwave irradiation). The reaction mixture was prepared as before and irradiated for 5 minutes at 300W in a CEM Discover Microwave. The treatment was the same as before.

$12 \mathrm{H}$-[1]Benzothieno[2,3- $\boldsymbol{d}$ ]pyrido[1,2-a]pyrimidin-12-one (5). Yellow solid. mp 237-239 ${ }^{\circ} \mathrm{C}$. ${ }^{1} \mathrm{H}$ NMR $\left(\mathrm{CDCl}_{3}\right) \delta 7.19(\mathrm{~d}, \mathrm{~J}=6.8 \mathrm{~Hz}, 1 \mathrm{H}), 7.45-7.60(\mathrm{~m}, 2 \mathrm{H}), 7.68-7.87(\mathrm{~m}, 3 \mathrm{H}), 8.71(\mathrm{~d}, \mathrm{~J}=$ $7.8 \mathrm{~Hz}, 1 \mathrm{H}), 9.25(\mathrm{~d}, \mathrm{~J}=6.8 \mathrm{~Hz}, 1 \mathrm{H}) .{ }^{13} \mathrm{C} \mathrm{NMR}\left(\mathrm{CDCl}_{3}\right): \delta 110.5(\mathrm{C}), 114.6(\mathrm{CH}), 122.2(\mathrm{CH})$, $124.5(\mathrm{CH}), 125.6(\mathrm{CH}), 125.9(\mathrm{CH}), 126.2(\mathrm{CH}), 127.4(\mathrm{CH}), 134.3(\mathrm{C}), 135.1(\mathrm{C}), 135.8(\mathrm{CH})$, 148.9 (C), 153.7 (C), 167.7 (C). IR (KBr) $1690(\mathrm{C}=\mathrm{O}) \mathrm{cm}^{-1}$. HRMS: $m / z$ calcd for $\mathrm{C}_{14} \mathrm{H}_{9} \mathrm{~N}_{2} \mathrm{OS}$ $\left[\mathrm{M}+\mathrm{H}^{+}\right]$253.04301. Found: 253.0430 .

8-Methyl-12H-[1]benzothieno[2,3- $d$ ]pyrido[1,2-a]pyrimidin-12-one (6). Yellow solid, mp 266-268 ${ }^{\circ} \mathrm{C} .{ }^{1} \mathrm{H}$ NMR $\left(\mathrm{CDCl}_{3}\right): \delta 2.53\left(\mathrm{~s}, 3 \mathrm{H}, \mathrm{CH}_{3}\right), 7.02(\mathrm{~d}, \mathrm{~J}=7.3 \mathrm{~Hz}, 1 \mathrm{H}), 7.43-7.58(\mathrm{~m}, 3 \mathrm{H})$, $7.83(\mathrm{~d}, \mathrm{~J}=8.0 \mathrm{~Hz}, 1 \mathrm{H}), 8.68(\mathrm{~d}, \mathrm{~J}=7.8 \mathrm{~Hz}, 1 \mathrm{H}), 9.14(\mathrm{~d}, \mathrm{~J}=7.3 \mathrm{~Hz}, 1 \mathrm{H}) .{ }^{13} \mathrm{C} \mathrm{NMR}\left(\mathrm{CDCl}_{3}\right): \delta$ $21.5\left(\mathrm{CH}_{3}\right), 109.7(\mathrm{C}), 117.3(\mathrm{CH}), 122.1(\mathrm{CH}), 123.5(\mathrm{CH}), 124.2(\mathrm{CH}), 125.7(\mathrm{CH}), 125.8$ (CH), $126.6(\mathrm{CH}), 134.4(\mathrm{C}), 134.8(\mathrm{C}), 148.1(\mathrm{C}), 148.9$ (C), 153.6 (C), 167.6 (C). IR (Nujol): $1698 \mathrm{~cm}^{-1}(\mathrm{C}=\mathrm{O})$. HRMS: $m / z$ calcd for $\mathrm{C}_{15} \mathrm{H}_{11} \mathrm{~N}_{2} \mathrm{OS}\left[\mathrm{M}+\mathrm{H}^{+}\right]$267.0587. Found: 267.0588 .

$8 \boldsymbol{H}$-[1]Benzothieno[2',3':4,5]pyrimido[2,1-a]isoquinolin-8-one (7). Yellow solid. mp 253$254^{\circ} \mathrm{C} .{ }^{1} \mathrm{H}$ NMR $\left(\mathrm{DMSO}_{\mathrm{d}}\right)$ ) $\delta$ 7.50-7.64 (m, 3H), 7.79-8.02 (m, 3H), $8.11(\mathrm{~d}, \mathrm{~J}=7.5 \mathrm{~Hz}, 1 \mathrm{H})$, $8.58(\mathrm{~d}, \mathrm{~J}=8.3 \mathrm{~Hz}, 1 \mathrm{H}), 8.89$ (d, $\mathrm{J}=7.8 \mathrm{~Hz}, 1 \mathrm{H}), 8.99$ (d, $\mathrm{J}=8.0 \mathrm{~Hz}, 1 \mathrm{H}) .{ }^{13} \mathrm{C}$ NMR (DMSO$\left.\mathrm{d}_{6}\right): \delta 112.1(\mathrm{C}), 114.4(\mathrm{CH}), 121.7(\mathrm{CH}), 122.1(\mathrm{CH}), 124.5(\mathrm{CH}), 125.7(\mathrm{CH}), 126.0(\mathrm{CH})$, $126.4(\mathrm{CH}), 127.3(\mathrm{CH}), 128.7(\mathrm{CH}), 131.4(\mathrm{C}), 132.5(\mathrm{CH}), 133.4(\mathrm{C}), 134.2(\mathrm{C}), 135.4(\mathrm{C})$, $147.6(\mathrm{C}), 154.4(\mathrm{C}), 166.3(\mathrm{C})$. IR (KBr): $1685(\mathrm{C}=\mathrm{O}) \mathrm{cm}^{-1}$. HRMS: $m / z$ calcd for $\mathrm{C}_{18} \mathrm{H}_{11} \mathrm{~N}_{2} \mathrm{OS}$ $\left[\mathrm{M}+\mathrm{H}^{+}\right]$303.0587. Found: 303.0589.

2-(2'-Methoxyphenyl)amino-3-carbomethoxybenzo-[b]-thiophene (8). Beige solid, mp 84$86^{\circ} \mathrm{C} .{ }^{1} \mathrm{H}$ NMR $\left(\mathrm{CDCl}_{3}\right): \delta 3.97\left(\mathrm{~s}, 3 \mathrm{H}, \mathrm{OCH}_{3}\right), 4.03\left(\mathrm{~s}, 3 \mathrm{H}, \mathrm{OCH}_{3}\right), 6.96-7.20(\mathrm{~m}, 4 \mathrm{H}), 7.36(\mathrm{~m}$, $1 \mathrm{H}), 7.59(\mathrm{~d}, \mathrm{~J}=7.9 \mathrm{~Hz}, 1 \mathrm{H}), 7.70(\mathrm{dd}, \mathrm{J}=7.7 \mathrm{~Hz}$ and $1.7 \mathrm{~Hz}, 1 \mathrm{H}), 8.18(\mathrm{~d}, \mathrm{~J}=8.2 \mathrm{~Hz}, 1 \mathrm{H})$, $10,86(\mathrm{~s}, 1 \mathrm{H}, \mathrm{NH}) .{ }^{13} \mathrm{C} \mathrm{NMR}\left(\mathrm{CDCl}_{3}\right): \delta 51.3\left(\mathrm{CH}_{3}\right), 56.0\left(\mathrm{CH}_{3}\right), 100.8(\mathrm{C}), 110.9(\mathrm{CH}), 117.6$ $(\mathrm{CH}), 120.7(\mathrm{CH}), 121.3(\mathrm{CH}), 122.4(2 \times \mathrm{CH}), 124.0(\mathrm{CH}), 125.7(\mathrm{CH}), 128.8(\mathrm{C}), 129.9(\mathrm{C})$, 136.5 (C), 149.8 (C), 160.6 (C), 167.2 (C). IR (KBr) $3181(\mathrm{NH}), 1654(\mathrm{C}=\mathrm{O}) \mathrm{cm}^{-1}$. HRMS: $\mathrm{m} / z$ calcd for $\mathrm{C}_{17} \mathrm{H}_{14} \mathrm{NO}_{3} \mathrm{~S}\left[\mathrm{M}-\mathrm{H}^{+}\right]$312.0689. Found: 312.0690 .

4-(4'-Methoxyphenyl)-2-phenylbenzo $[b]$ thieno[2,3-d]pyrimidine (9). Yellow solid, mp 203$204^{\circ} \mathrm{C} .{ }^{1} \mathrm{H}$ NMR $\left(\mathrm{CDCl}_{3}\right) \delta 3.86\left(\mathrm{~s}, 3 \mathrm{H}, \mathrm{OCH}_{3}\right), 7.04(\mathrm{dd}, \mathrm{J}=6.7 \mathrm{~Hz}$ and $2.1 \mathrm{~Hz}, 2 \mathrm{H}), 7.15-7.22$ $(\mathrm{m}, 1 \mathrm{H}), 7.34-7.45(\mathrm{~m}, 4 \mathrm{H}), 7.66-7.83(\mathrm{~m}, 4 \mathrm{H}), 8.52-8.58(\mathrm{~m}, 2 \mathrm{H}) .{ }^{13} \mathrm{C} \mathrm{NMR}\left(\mathrm{CDCl}_{3}\right): \delta 55.5$ $\left(\mathrm{CH}_{3}\right), 114.2(2 \times \mathrm{XH}), 122.5(\mathrm{C}), 123.0(\mathrm{CH}), 124.8(\mathrm{CH}), 124.9(\mathrm{CH}), 127.6(\mathrm{CH}), 128.5$ $(2 \times C H), 128.6(2 \times C H), 130.6(\mathrm{CH}), 130.7(2 \times C H), 131.0(\mathrm{C}), 131.8(\mathrm{C}), 137.1(\mathrm{C}), 137.4(\mathrm{C})$, 160.7 (C), 161.2 (C), 161.6 (C), 171.7 (C). HRMS: $m / z$ calcd for $\mathrm{C}_{23} \mathrm{H}_{17} \mathrm{~N}_{2} \mathrm{OS}\left[\mathrm{M}+\mathrm{H}^{+}\right]$369.1056. Found: 369.1049 . 


\section{References}

1. Modica, M.; Santagati, M.; Santagati, A.; Cutuli, V.; Mangano, N.; Caruso, A. Pharmazie 2000, 55, 500 .

2. (a) El-Sherbeny, M. A.; El-Ashmawy, M. B.; El-Subbagh, H. I.; El-Emam, A. A.; Badria, F. A. Eur. J. Med. Chem. 1995, 30, 445. (b) Chambhare, R. V.; Khadse, B. G.; Bobde, A. S.; Bahekar, R. H. Eur. J. Med. Chem. 2003, 38, 89.

3. (a) Perrissin, M.; Favre, M.; Luu-Duc, C.; Bakri-Logeais, F.; Huguet, F.; Narcisse, G. Eur. J. Med. Chem. Chim. Ther. 1984, 5, 420. (b) Alagarsamy, V.; Meena, S.; Ramseshu, K. V.; Solomon, V. R.; Thirumurugan, K.; Dhanabal, K.; Murugan, M. Eur. J. Med. Chem. 2006, 41, 1293.

4. (a) Wang, Y. D.; Johnson, S.; Powell, D.; McGinnis, J. P.; Miranda, M.; Rabindran, S. K. Bioorg. Med. Chem. Lett. 2005, 15, 3763. (b) Jennings, L. D.; Kincaid, S. L.; Wang, Y. D.; Krishnamurthy, G.; Beyer, C. F.; McGinnis, J. P.; Miranda, M.; Discafani, C. M.; Rabindran, S. K. Bioorg. Med. Chem. Lett. 2005, 15, 4731.

5. Munchhof, M. J.; Beebe, J. S.; Casavant, J. M.; Cooper, B. A.; Doty, J. L.; Higdon, R. C.; Hillerman, S. M.; Soderstrom, C. I.; Knauth, E. A.; Marx, M. A.; Rossi, A. M. K.; Sobolov, S. B.; Sun, J. Bioorg. Med. Chem. Lett. 2004, 14, 21.

6. Meyer, M. D.; Altenbach, R. J.; Basha, F. Z.; Carroll, W. A.; Condon, S.; Elmore, S. W.; Kerwin, J. F.; Sippy, K. B.; Tietje, K.; Wendt, M. D.; Hancock, A.; Brune, M. E.; Buckner, S. A.; Drizin, I. J. Med. Chem. 2000, 43, 1586.

7. (a) Zheng, G. Z.; Bhatia, P.; Daanen, J.; Kolasa, T.; Patel, M.; Latshaw, S.; El Kouhen, O. F.; Chang, R.; Uchic, M. E.; Miller, L.; Nakane, M.; Lehto, S. G.; Honore, M. P.; Moreland, R. B.; Brioni, J. D.; Stewart, A. O. J. Med. Chem. 2005, 48, 7374. (b) Zheng, G. Z.; Bhatia, P.; Kolasa, T.; Patel, M.; El Kouhen, O. F.; Chang, R.; Uchic, M. E.; Miller, L.; Baker, S.; Lehto, S. G.; Honore, M. P.; Wetter, J. M.; Marsh, K. C.; Moreland, R. B.; Brioni, J. D.; Stewart, A. O. Bioorg. Med. Chem. Lett. 2006, 16, 4936.

8. Begouin, A.; Hesse, S.; Queiroz, M.-J. R. P.; Kirsch, G. Synthesis 2006, 2794.

9. Ihmels, H.; Faulhaber, K.; Viola, G. Evaluation of the DNA-binding properties of cationic dyes by absorption and emission spectroscopy, In Schmuck, C.; Wennemers, H.; Eds.) Highlights in Bioorganic Chemistry: Methods and Applications; Wiley VCH: 2004; pp 172187.

10. Viola, G.; Salvador, A.; Vedaldi, D.; Disaro, E. F. S.; Basso, G.; Queiroz M.-J. R. P. Journal of Photochemistry and Photobiology B: Biology 2006, 82, 105.

11. (a) Prim, D.; Joseph, D.; Kirsch, G. Liebigs Ann. 1996, 239. (b) Joseph, D.; Martarello, L.; Kirsch, G. J. Chem. Research (S) 1995, 448. (c) Martarello, L.; Joseph, D.; Kirsch, G. Heterocycles 1996, 43, 367.

12. Romagnoli, R.; Baraldi, P. G.; Moorman, A. R.; Iaconinoto, M. A.; Carrion, M. D.; Cara, C. L.; Tabrizi, M. A.; Preti, D.; Fruttarolo, F.; Baker, S.; Varani, K.; Borea, P. A. Bioorg. Med. Chem. Lett. 2006, 5530.

13. Pla, D.; Marchal, A.; Olsen, C. A.; Albericio, F.; Alvarez, M. J. Org. Chem. 2005, 70, 8231.

14. De La Hoz, A.; Diaz-Ortiz, A.; Mateo, M. C.; Moral, M.; Moreno, A.; Elguero, J.; FocesFoces, C.; Rodrigues, M. L.; Sanchez-Migallon, A. Tetrahedron 2006, 5868. 